\title{
Determination of the isotopic composition of an enriched hafnium spike by MC-ICP-MS using regression model
}

\author{
RAn Lin Jie Lin KeQIng Zong KAng Chen \\ ${ }^{1}$ School of Earth Sciences, China University of Geosciences, \\ Wuhan 430074, China
}

Isotope dilution (ID) method, owing to its high precision, is extensively applied for the determination of element concentrations in a wide range of natural samples. One of the prerequisites of ID method is that isotopic compositions of an enriched spike and sample need to be known, but the determination of isotopic composition of the enriched spike is challenging. In this study, a regression mass bias correction model is employed for the accurate and precise determination of the isotopic composition of an enriched spike Hf (Lot No. 159293) by using of NRC IRIS-1 Ir and NIST SRM 3134 Re isotopic standard. The determined isotope ratios of ${ }^{176} \mathrm{Hf} /{ }^{177} \mathrm{Hf}$, ${ }^{179} \mathrm{Hf} /{ }^{177} \mathrm{Hf}$ and ${ }^{180} \mathrm{Hf} /{ }^{177} \mathrm{Hf}$ of the spike $\mathrm{Hf}$ are $0.2406(5)_{k=1}, 2.8620(5)_{k=1}$ and 384.65(1) $)_{k=1}$, respectively, which meet the required precision levels of $\mathrm{Hf}$ isotopes in the application of the ID method. The combined uncertainty estimations for the calibrated Re and $\mathrm{Hf}$ isotope ratios are evaluated using Monte Carlo Method with consideration of the uncertainty of the primary calibrator (IRIS-1, ${ }^{193} \mathrm{Ir} /{ }^{191} \mathrm{Ir}=1.6866 \pm 0.0005, u, k=1$ ). To the best of our knowledge, this is first report of calibrating the isotope ratios in a spike using the regression model. The accurately calibrated spike is anticipated to reduce the uncertainty for measuring the mass fraction of Hf significantly, thereby increasing the accuracy of the Lu-Hf chronometer. 